\title{
Image Analysis of 2D X-ray Intensity Maps: Element Abundances, Mineralogy, and Modal Analysis of Meteorites
}

\author{
Denton S. Ebel ${ }^{1,2}$, Ellen J. Crapster-Pregont ${ }^{1,2}$, and Jon M. Friedrich ${ }^{1,3}$ \\ ${ }^{1}$ Dept. of Earth and Planetary Sciences, American Museum of Natural History, New York, NY 10024. \\ 2.Lamont-Doherty Earth Observatory, Columbia University, Palisades, NY 10964. \\ ${ }^{3}$ Department of Chemistry, Fordham University, Bronx, NY 10458.
}

X-ray mapping is increasingly used in the geological sciences for qualitative observations of textures, mineral zoning, and element distribution among rock components [1-4]. We combine mapping with an aggressive program of image analysis in deepening our understanding of the early solar system through the analysis of chondritic meteorites [5,6]. Chondrites are "sediments" that accreted in the earliest solar system into planetesimals now represented by objects in the asteroid belt. They accumulated hightemperature inclusions (igneous rock droplets or aggregations) with a low-temperature fine-grained mineral matrix including pre-solar grains and macromolecular carbon [7]. We seek to measure the relative abundances, modal mineralogies, and chemical compositions of these components with such different thermal histories, to higher accuracy than has been done previously.

Here, we present our optimization of information return from mapping through recovery of quantitative results. Results yield first order major element bulk compositions and phase abundances of chondrules and other inclusions in meteorites, and the mean composition of matrix. The trick is to do this with minimal time on maximum sample area. We are implementing and adapting techniques explored by previous workers [e.g., 4]. In some chondrites, inclusions average $\sim 150 \mu \mathrm{m}$ diameter, in others, $d>500$ $\mu \mathrm{m}$. Thus spatial resolution and powerful statistical sampling of inclusions are tightly coupled [6].

We use the AMNH 5-spectrometer (wavelength dispersive, WDS) CAMECA SX100 electron microprobe with BrukerNano energy dispersive spectrometer (EDS) to map large areas at various spatial resolutions (1-13 $\mu \mathrm{m} /$ pixel) at $15 \mathrm{keV}$ accelerating voltage, with a $1 \mu \mathrm{m}$ spot, a perpendicular beam and rastering exclusively by stage motion. Typically we make a single pass, without backgrounds, on $\mathrm{Mg}$, $\mathrm{Ni}, \mathrm{Ti}, \mathrm{Al}, \mathrm{Ca}$ (WDS) and S, Si, Fe (EDS). The CAMECA system currently allows two more elements on EDS than in our earlier work. Tens of maps (32-bit tiff output to avoid scaling effects [2]) are stitched together offline using custom software written in IDL. The tradeoff between area mapped and count rates requires careful choices of beam current and dwell time. Filament life would be compromised by the use of high current, so currents $\leq 40 \mathrm{nA}$ are used. High counts/pixel are necessary to apply a full correction for matrix effects [1], but this limits map area due to constraints on instrument time. Our method is most similar to [4], who noted that backgrounds are lower for short dwell time [8].

Calibration of counts to $\mathrm{wt} \%$ correction, and assignment of background count rates, is done by mapping standards. Raw count/sec/nA on standards are close to linear with composition, and element counts on several standards lacking an element yield a background curve for that element as a function of standard mean atomic mass $(Z)$. We are aided by the fact that the suite of minerals common in meteorites is much smaller than in terrestrial rocks [9]. Atomic number (Z), absorption (A), and fluorescence (F) corrections are negligible for most of the samples considered here. $\mathrm{Z}$ for most minerals found in carbonaceous chondrites are in a narrow range. Corrections $\mathrm{Z}$ and $\mathrm{A}$ are only problematic in FeO-rich 
silicates. Due to low current (20nA) and relatively short dwell times, F also does not greatly affect the conversion of intensity to concentration.

Chondritic meteorites consist of mineral \pm quenched silicate liquid (glass) inclusions (e.g., liquid droplet chondrules) surrounded by extremely fine-grained matrix [7, 10-13]. Image analysis begins with manual outlining (segmentation) of inclusions and categorizing them by type in a drawing program. Stacking of registered (aligned) element and RGB mosaic maps facilitates this outlining allowing for the identification of inclusion boundaries based on observation of multiple stacked composition maps. The diversity of inclusion types and chemistry has foiled attempts to automate segmentation, which appears to be possible, if to a limited extent, with good maps of $\mathrm{Na}$ [14]. Typically, we obtain single maps of polished thin or thick sections with over 1000 inclusions, which begin to be representative of bulk.

Segmented maps are coded for inclusion type using grayscales. Imported into ImageJ and thresholded, the center of mass of each inclusion may be output to a table. This table, plus the grayscale map, allows identification of every pixel that belongs in each inclusion using custom software. By assigning a single RGB color to each inclusion, a new map is created which serves to uniquely identify the pixels in each inclusion. The RGB color and min and max $x-y$ coordinates of each inclusion are added to the table. With this table and the RGB inclusion map, pixel-by-pixel analysis of very large numbers of inclusions across all the $\mathrm{x}$-ray maps and to rapidly change parameters to re-run this analysis.

The derived data (inclusion map and table) and all the x-ray maps are loaded into arrays in custom IDL software. Determination of modal abundances of inclusion types and matrix abundance is straightforward. We also obtain a bulk chemical analysis of every inclusion, based on hundreds or more pixels of data in each one [15], by simply calculating the mean intensity of each element per pixel. We have employed several approaches to determining the mineralogical identity of each pixel. Beginning with the simplest minerals (e.g., Fe-Ni metal, $\mathrm{MgAl}_{2} \mathrm{O}_{4}$ spinel, FeS troilite) we can query each x-ray map or linear combinations of maps on a pixel-by-pixel basis. This approach yields mineral modal abundance data for each inclusion. We are combining this data with 3D CT scan data on inclusion sizes and spatial relationships. We welcome commentary regarding the robustness of our methods.

\section{References:}

[1] GL Clarke et al., Journal of Metamorphic Geology 19 (2001), p. 635-644

[2] JJ Freil and CE Lyman, Microscopy and Microanalysis 12 (2006), p. 2-25.

[3] V de Andrade et al., Journal of Metamorphic Geology 24 (2006), p. 655-668.

[4] D Prêt et al., American Mineralogist 95 (2010), p. 1379-1388.

[5] DS Ebel et al., Microscopy and Microanalysis 19, Supp. S2 (2013), p. 632-633.

[6] EJ Crapster-Pregont and DS Ebel, this meeting (2014).

[7] AJ Brearley \& R Jones, In Planetary Materials (JJ Papike, ed.) pp 3-1 - 3-398, MSA Rev. Min. 36.

[8] JI Goldstein et al., Scanning Electron Microscopy and X-ray Microanalysis, 2nd Edition. Plenum, NY (1992) 820 pp.

[9] RM Hazen et al., American Mineralogist 93 (2008), p.1693-1720.

[10] JM Bayron et al., Lunar and Planetary Science XLV (2014) Abs. \#1225.

[11] A Lobo et al., Lunar and Planetary Science XLV (2014) Abs. \#1423.

[12] EJ Crapster-Pregont and DS Ebel, Lunar and Planetary Science XLV (2014) Abs. \#1379.

[13] DS Ebel et al., Lunar and Planetary Science XXXIX (2014) Abs. \#2121.

[14] JN Grossman \& AJ Brearley, Meteoritics and Planetary Science 40 (2005), p. 87-122.

[15] DS Ebel et al., Lunar and Planetary Science XXXIX (2014) Abs. \#1206. 